PERSPECTIVE OPEN

\title{
An algorithm recommendation for the pharmacological management of allergic rhinitis in the UK: a consensus statement from an expert panel
}

\author{
Brian Lipworth, Jon Newton ${ }^{2}$, Bhaskar Ram ${ }^{3}$, lain Small ${ }^{4}$ and Jürgen Schwarze ${ }^{5}$
}

\begin{abstract}
Allergic rhinitis is a frequent presenting problem in primary care in the UK, and has increased in prevalence over the last 30 years. When symptomatic, patients report significant reduction in their quality of life and impairment in school and work performance. Achieving adequate symptom control is pivotal to successful allergic rhinitis management, and relies mostly on pharmacotherapy. While it is recognised that most mild-moderate allergic rhinitis symptoms can be managed successfully in primary care, important gaps in general practitioner training in relation to allergic rhinitis have been identified. With the availability of new effective combination therapies, such as the novel intranasal formulation of azelastine hydrochloride and fluticasone propionate in a single device (Dymista ${ }^{\oplus}$; Meda), the majority of allergic rhinitis symptoms can be treated in the primary care setting. The primary objective of this consensus statement is to improve diagnosis and treatment of allergic rhinitis in primary care, and offer guidance on appropriate referral of difficult-to-treat patients into secondary care. The guidance provided herein outlines a sequential treatment pathway for allergic rhinitis in primary care that incorporates a considered approach to improve the management of allergic rhinitis symptoms and improve compliance and patient satisfaction with therapy. Adherence with this care pathway has the potential to limit the cost of providing effective allergic rhinitis management in the UK by avoiding unnecessary treatments and investigations, and avoiding the need for costly referrals to secondary care in the majority of allergic rhinitis cases. The fundamentals presented in this consensus article should apply in most health-care settings.
\end{abstract}

npj Primary Care Respiratory Medicine (2017)27:3 ; doi:10.1038/s41533-016-0001-y

\section{INTRODUCTION}

The aim of any disease management plan is control of the disease. In 2015, a multi-professional group of clinicians met in Stirling, Scotland to discuss the development of a simplified allergic rhinitis (AR) treatment algorithm for use in primary and secondary care, incorporating current knowledge and recently available treatments. This consensus statement is a result of that initial meeting and subsequent collaboration among the working party. The primary objective is to promote better management of AR patients, improve diagnosis and treatment in primary care and to facilitate transition of difficult-to-treat patients into secondary care.

\section{HOW AR CAN AFFECT PATIENTS?}

AR a common condition that affects $>20 \%$ of the UK population, is a frequent presenting problem in primary care, ${ }^{1,2}$ and is increasing in prevalence., ${ }^{3,4}$ It is associated with considerable morbidity; significantly reducing quality of life, ${ }^{5,6}$ interfering with attendance and performance at school and work, ${ }^{7-9}$ and resulting in considerable health-care and indirect costs. ${ }^{10-13}$ Around $50-80 \%$ of AR patients report that their condition interferes with sleep, resulting in daytime fatigue, decreased alertness, inability to concentrate, depression and irritability. ${ }^{14} \mathrm{AR}$ is also associated with a number of coexisting conditions, including asthma, sinusitis, upper respiratory tract infection, otitis media with effusion and nasal polyposis. ${ }^{15,16}$

\section{AR MANAGEMENT IN THE UK}

Management of AR is generally not allergen-specific and relies in the main on pharmacotherapy to control symptoms. ${ }^{17,18}$ Most mild-moderate AR symptoms can be managed successfully in primary care. However, one-third of general practitioner (GP) specialty training programmes do not provide allergy training. ${ }^{19}$ Initial AR monotherapy is failing many patients in the UK. ${ }^{20} \mathrm{~A}$ database survey of 22,000 AR patients living in the UK showed that the majority of seasonal AR (SAR) and perennial AR (PAR) patients received monotherapy as their initial prescription of the season, but such monotherapy (e.g., antihistamine $[\mathrm{AH}]$ or intranasal corticosteroid [INCS]) proved insufficient for many (20-25\% SAR patients; $37-46 \%$ of PAR patients). Other patients started the season on a multi-therapy regimen $33 \%$ and $23 \%$ of SAR and PAR patients, respectively) and this proportion increased during the season. As the number of therapies prescribed increased, so too did the number of return GP consultations. By

\footnotetext{
${ }^{1}$ Ninewells Hospital and Medical School, Scottish Centre for Respiratory Research, Dundee, Scotland, UK; ${ }^{2}$ Forth Valley Royal Hospital, Forth Valley Health Board, Larbert, Scotland, UK; ${ }^{3}$ Aberdeen Royal Infirmary, Grampian Health Board, Aberdeen, Scotland, UK; ${ }^{4}$ Peterhead Surgery, Grampian Health Board, Peterhead, Scotland, UK and ${ }^{5}$ Child Life and Health, The University of Edinburgh, Edinburgh, Scotland, UK

Correspondence: Brian Lipworth (b.j.lipworth@dundee.ac.uk)
}

Received: 24 November 2015 Revised: 5 September 2016 Accepted: 20 October 2016

Published online: 23 January 2017 
the end of the season around half of all AR sufferers were prescribed multiple therapies, most commonly oral AH plus INCS. However, this combination is not supported by clinical trials of combination regimens vs. monotherapy. ${ }^{21,22}$ The Allergic Rhinitis and its Impact on Asthma (ARIA) guidelines state that "combination between drugs has been tested, but insufficient data are available to make a recommendation concerning the combined use of oral $\mathrm{AH}$ and INCS". 23

\section{WHY IT'S IMPORTANT TO GET RHINITIS UNDER CONTROL}

The link between AR and asthma is well-established. ${ }^{23,24}$ Both AR and non-AR are risk factors for the development of asthma; comorbid rhinitis affects up to $75 \%$ of those with asthma. ${ }^{24,25}$ Poor control of rhinitis predicts poor control of asthma; asthma patients with significant rhinitis are four-times more likely to have poorlycontrolled asthma than those without, ${ }^{24}$ with a negative impact on asthma control equivalent to that of smoking. ${ }^{26}$ Moreover, failure to treat $A R$ appropriately results in increased asthma medication use for co-morbid patients. ${ }^{27}$

\section{ASSESSMENT OF AR CONTROL}

Some sophisticated AR control questionnaires have been developed including the Control of Allergic Rhinitis and Asthma Test ${ }^{28}$ and Rhinitis Control Assessment Test. ${ }^{29,30}$ Others advocate a multifactorial assessment incorporating an assessment of (i) severity and/or frequency of daily or nocturnal symptoms, (ii) impairments in social, physical, professional and educational activities, (iii) respiratory function monitoring and (iv) exacerbations (e.g., unscheduled medical consultations and rescue medication use). ${ }^{31}$ Although useful in clinical trials these are unlikely to be used routinely in day-to-day practice. ARIA has recently recommended a simple visual analogue scale (VAS) as the new language of AR control, with a score of $5 / 10 \mathrm{~cm}$ used to assess control and guide treatment decisions as part of a simple algorithm called the AR clinical decisions support system (CDSS). ${ }^{32}$ This VAS has also been incorporated into an app for patients (Allery Diary). A companion app for physicians is currently under development (which includes the AR CDSS; Allergy Diary Companion), thus linking key stakeholders with a common language of AR control.

\section{WHAT AR PATIENTS WANT FROM TREATMENT}

Living with symptomatic AR means coping with any or all of the symptoms of nasal congestion, headache, postnasal drip, repeated sneezing, runny nose and other symptoms on a near-daily basis. ${ }^{33}$ AR patients have high expectations from their treatment, ${ }^{34}$ but also a high degree of dissatisfaction with AR treatment, ${ }^{35}$ for reasons that include: symptom breakthrough, lack of 24-hour coverage, lack of coverage of both nasal and ocular symptoms, and unwanted side effects. ${ }^{15}$ Patients want faster and more complete symptom control. ${ }^{27}$ Insufficient symptom control with INCS and oral AH is a major concern, and a situation, which has not improved over time. ${ }^{15}$ Many physicians underestimate AR severity and consequently fail to issue adequate treatment, ${ }^{36}$ a situation which appears to have changed little in a decade. ${ }^{37}$

\section{MANAGEMENT IN PRIMARY CARE-DIAGNOSIS}

The definition, aetiology and classifications of AR are described in Table $1,{ }^{1,23}$ and the diagnosis of AR by symptom assessment is outlined in Fig. 1. ${ }^{23}$ Confirmation of the diagnosis may be made through questions concerning family history, social history (housing, pets, occupation, possible triggers), and visual examination. The common triggers for AR are listed in Table $1 .{ }^{1}$

$A R$ is the predominant form of rhinitis in children, but also accounts for one-third of adult cases. ${ }^{1}$ At greatest risk are those with a personal or family history of atopy (defined by positive skin prick tests or specific lgE to common aeroallergens). ${ }^{2}$ The clinical history should determine whether allergy testing is required, which may be useful to identify or exclude an allergic trigger that may influence management, e.g., aeroallergen avoidance. GPs should perform either skin prick testing or serum total/specific lgE depending on local availability. The former has the advantage that it is cheaper and the patient gets instant feedback, but is time consuming and requires stopping anti-histamines for a week. Ideally, allergy testing would be performed in all patients with rhinitis not only for advice about potential allergen avoidance but also because it will alter the management pathway. Allergy testing will not, however, identify non antigenic chemical irritants which can cause nasal airway hyper-reactivity through a non lgEmediated pathway. Indeed in many cases exogenous chemical irritants may aggravate underlying AR. In reality, the majority of patients presenting in primary care with $A R$ can be managed without formal identification of the specific allergic trigger ${ }^{18}$ with pharmacotherapy.

\section{MANAGEMENT IN PRIMARY CARE-TREATMENT}

Figure 2a shows an algorithm for the treatment of AR in primary care, adapted by the consensus group from the 2008 British Society of Allergy \& Clinical Immunology (BSACl) guidelines ${ }^{1}$ in order to simplify treatment in primary care and incorporate recently available treatment options.

\section{Education}

Patients should be educated on the nature of AR, allergen avoidance, available treatments (including safety and potential side effects), and be given realistic expectations of the results of therapy. Treatment failure may be related to poor technique in the use of nasal sprays and drops and, therefore, appropriate training is essential.

\section{Treatment options}

Nasal douching. Saline douching is a safe, inexpensive treatment, demonstrated to reduce symptoms in children and adults with seasonal rhinitis. ${ }^{38,39}$ It is more commonly used in mainland Europe than in the UK. In our opinion it has only a limited role in the management of $A R$, and there is little objective data to support its use.

Oral/topical non-sedating antihistamines. Oral and topical nonsedating AHs are recommended as first-line therapy for mildmoderate intermittent and mild persistent AR. The therapeutic effect of intranasal $\mathrm{AH}$ is superior to that that of oral $\mathrm{AH}^{40}$ with the additional benefit of a faster onset of action (i.e., within 15 mins). ${ }^{41}$ Patients responsive to $\mathrm{AH}$ therapy should be instructed to continue therapy during periods of allergen exposure. Those non-responsive to therapy (e.g., symptomatic after 2 weeks) should be stepped up.

Intranasal corticosteroids. INCSs are recommended as first-line therapy for moderate-severe persistent symptoms and treatment failures with AHs alone. For patients who do not tolerate INCSs or prefer not to use them, adding a leukotriene receptor antagonist to an oral or topical $\mathrm{AH}$ may be considered. ${ }^{42,43}$ Responsive patients should be instructed to continue therapy during periods of allergen exposure. Those non-responsive to therapy (e.g., symptomatic after 4 weeks) should be stepped up.

Recently-approved AR treatment. The combination of intranasal azelastine hydrochloride (AZE) and fluticasone propionate (FP) in a single device (Dymista ${ }^{\circledR}$ (INCS/AZE); Meda, Solna, Sweden) is a novel formulation that is indicated for adults and adolescents $\geq 12$ years with moderate/severe SAR or PAR if monotherapy with 


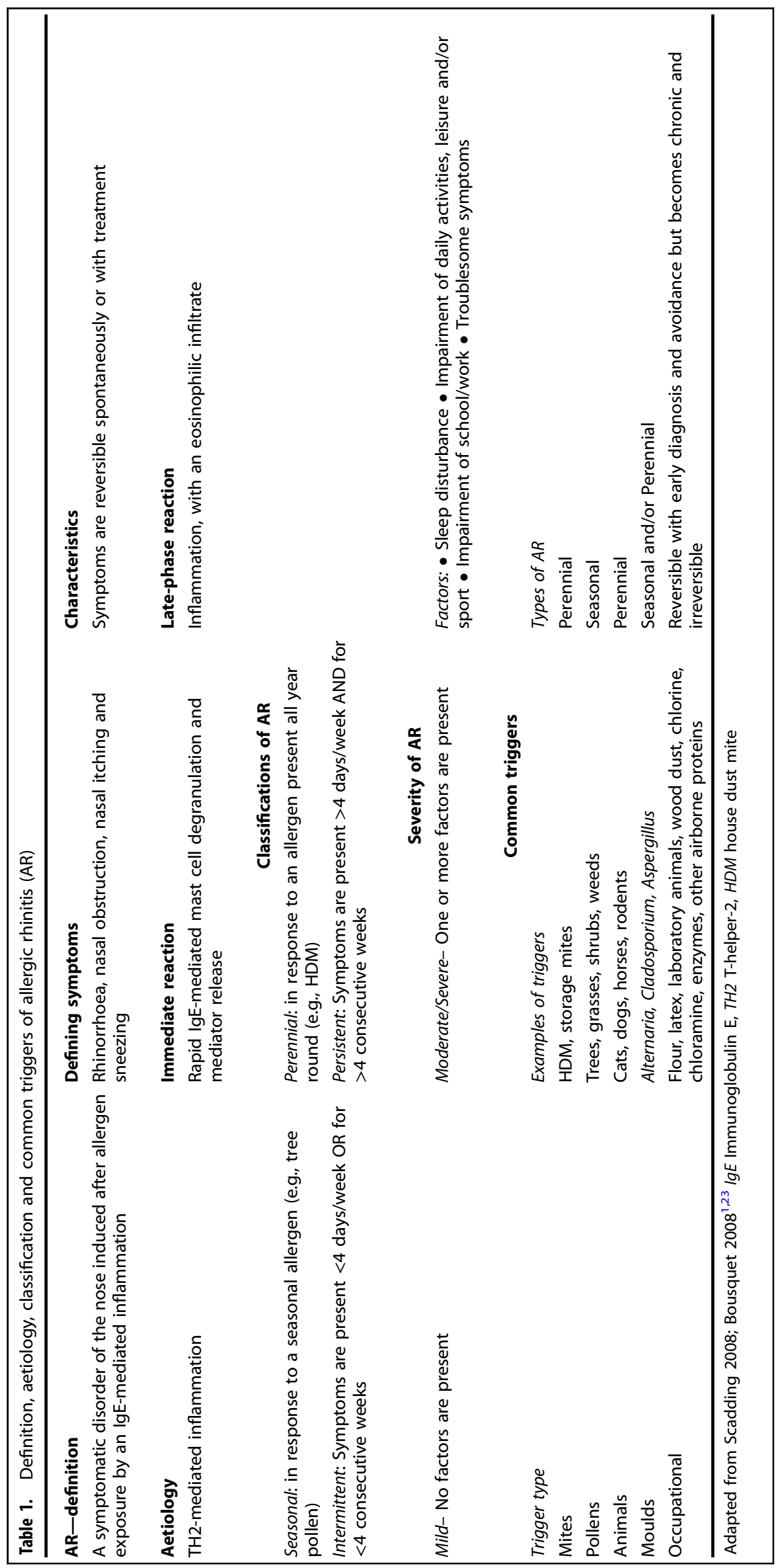



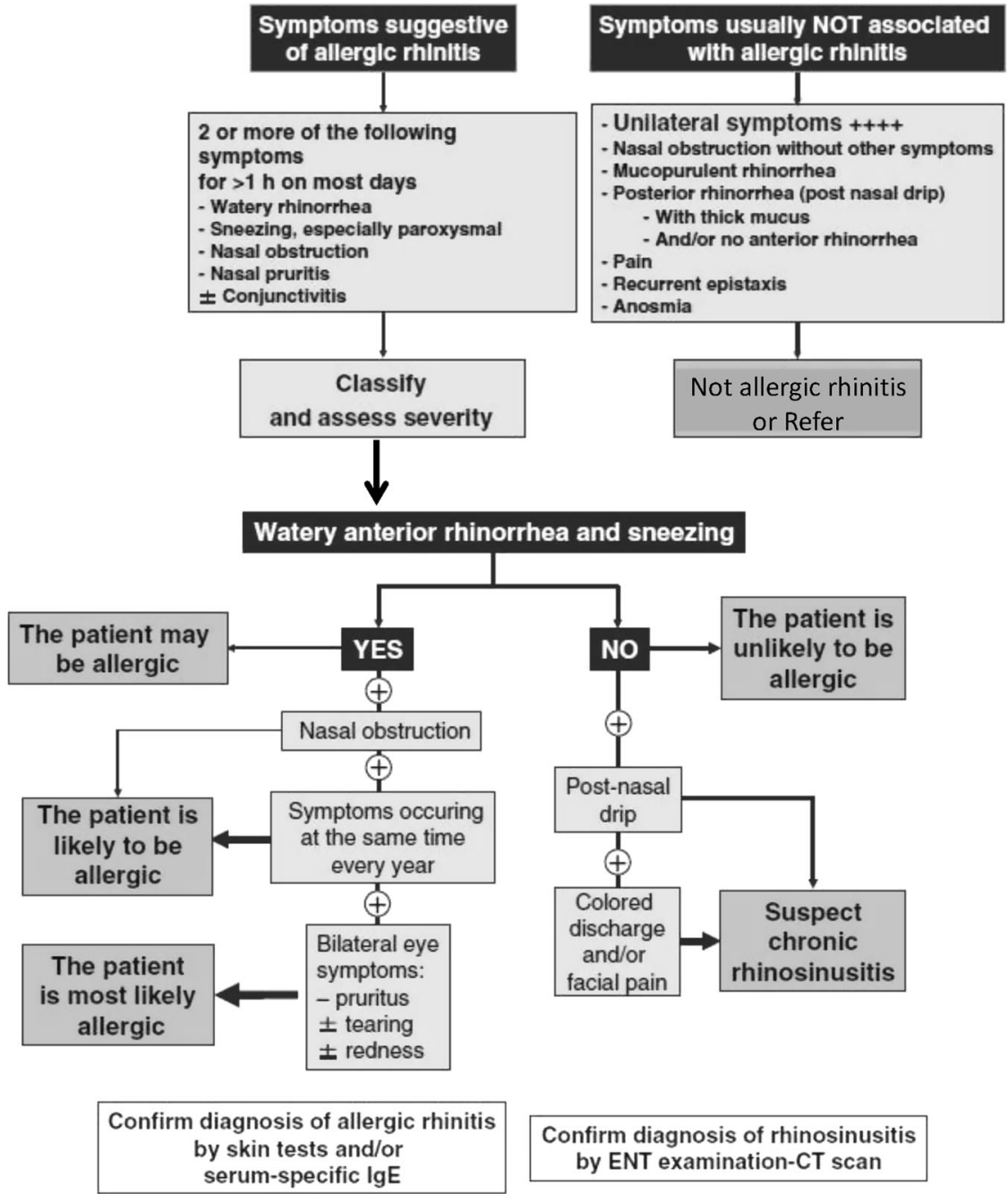

Fig. 1 Diagnosis of allergic rhinitis through symptom assessment. Adapted from ARIA Bousquet 2008. ${ }^{23}$ IgE: immunoglobulin E; ENT: Ear Nose Throat; CT: computed tomography

either INCS or AH is not considered sufficient. ${ }^{44}$ In primary care, the consensus group considers that INCS/AZE (single device) may be used second line after failure of topical INCS after checking for the correct use and compliance with medication. If incorrect use or non-compliance is found, re-education followed by repeat trial of topical INCS is recommended before INCS/AZE (single device) prescription. Alternatively, the addition of an oral AH to INCS may be considered in the case of INCS monotherapy failure, particularly for children $<12$ years for whom INCS/AZE (single device) is not yet indicated. However, it should be noted that the evidence to support this practice is lacking. ${ }^{21,45}$

INCS/AZE (single device) may be used first line in certain cases in which the GP considers that monotherapy is more likely to fail, if they consider it warranted in their professional opinion, in accordance with the labelled product indication. ${ }^{44}$ This recommendation is based on an extensive review of existing clinical trial evidence, ${ }^{46-49}$ and data obtained in routine clinical practice in several countries in Europe including Germany, Sweden, Denmark, Norway and Romania. ${ }^{50}$ INCS/AZE (single device) is recommended first-line in the recently published AR CDSS from ARIA for those patients with a VAS score $>5 / 10 \mathrm{~cm} .{ }^{32}$ The speed of onset with INCS/AZE (single device) for both nasal and ocular symptom relief and the use of a single spray may improve compliance and treatment outcomes by avoiding administration and delivery issues. ${ }^{51}$
Oral corticosteroids. This consensus group does not recommend routine early use of oral corticosteroids (OC), which should be reserved for short-term acute severe symptoms, in combination with a topical INCS or AZE/INCS (single device). A suggested regime for adults is $0.5 \mathrm{mg} / \mathrm{kg}$ given orally in the morning for 5-10 days. ${ }^{1}$

Referral

In the event of treatment failure on second-line INCS/AZE (single device) in primary care, checking for the correct use of medication, compliance, and whether the correct diagnosis was made is advised. Following that, referral to secondary care is recommended for further investigations.

Management in secondary care

In secondary care, the consensus group considers that INCS/AZE (single device) could be used as a first-line alternative to topical INCS in patients with moderate-severe symptoms (Fig. 2b). This is in contrast to primary care where high bioavailability INCS (e.g., beclomethasone dipropionate) first line is often prescribed based on formulary recommendation and acquisition cost rather than on efficacy, safety or patient-reported outcomes. The consensus group, however, had a preference for modern, low bioavailability 
steroids (e.g., budesonide; FP, fluticasone furoate or mometasone furoate).

Symptom-specific investigations for refractory symptoms should be completed in secondary care following INCS/AZE (single device). Add-on therapy with symptom-specific treatment such as ipratropium for watery rhinorrhoea, or oral $\mathrm{AH}$ for nasal itching/sneezing may be reserved for use in secondary care. It should be noted that currently there is no scientific evidence to support this practice. ${ }^{21,45}$ In the event of INCS/AZE (single device) failure, the consensus group recommended adding $O C$ for

\section{A: primary care}

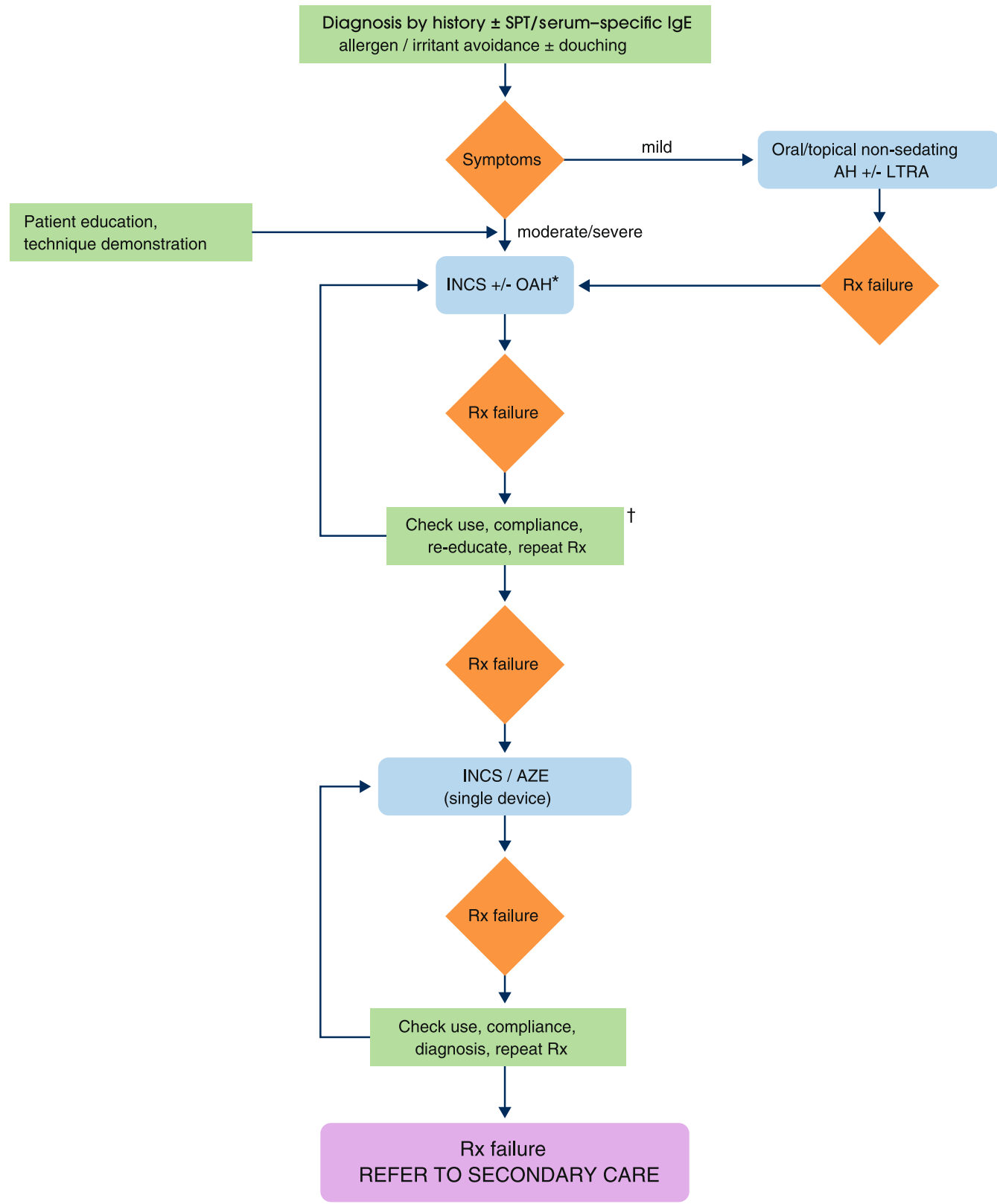




\section{B: secondary care}

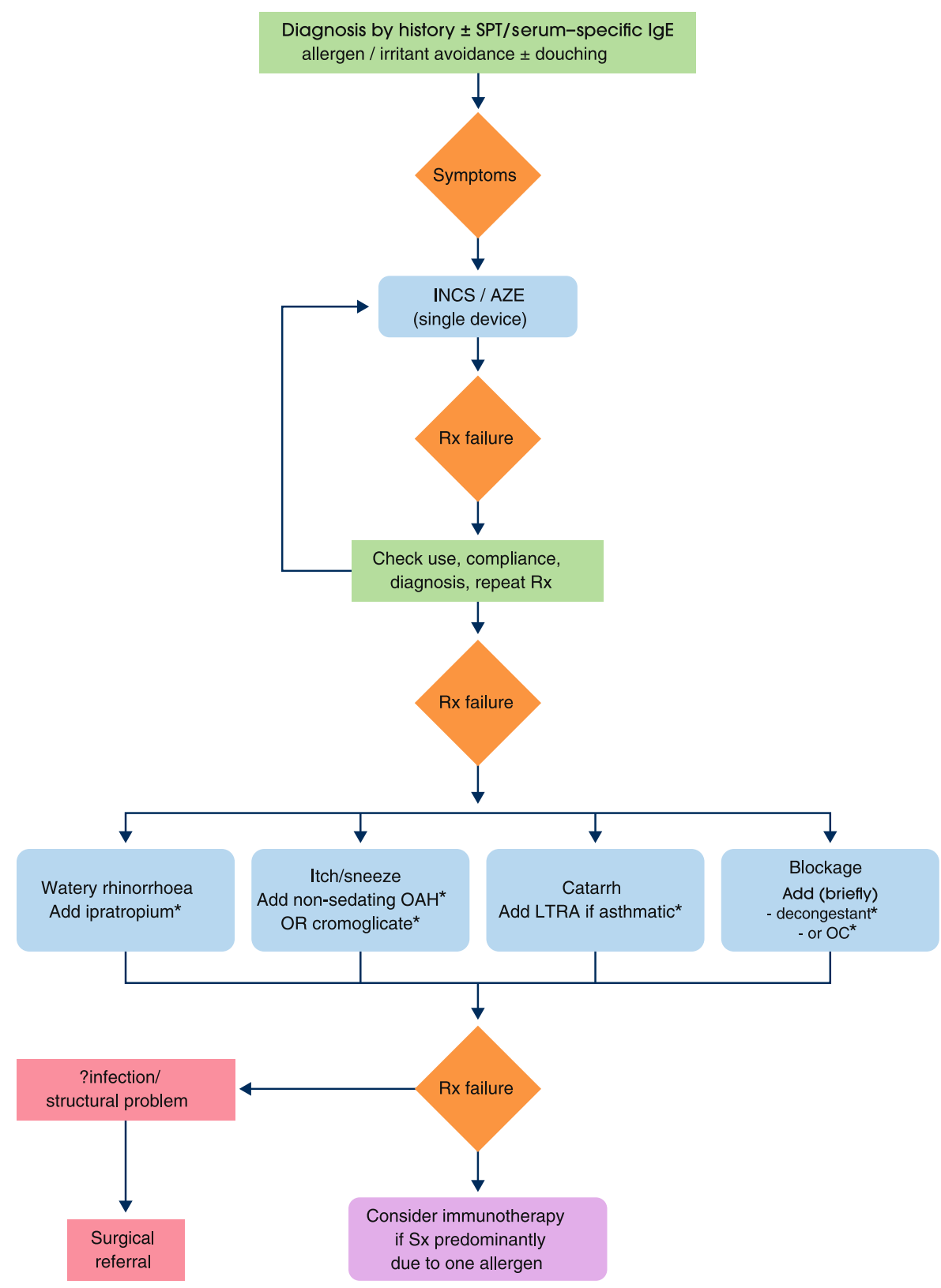

Fig. 2 Treatment algorithm for allergic rhinitis in (a) primary care and (b) secondary care. AH: anti-histamine; AZE: azelastine; IgE: immunoglobulin E; INCS: intranasal corticosteroid; LTRA: leukotriene receptor antagonist; OAH: oral antihistamine; OC: oral corticosteroid; Rx: treatment; SPT: skin prick test; Sx: symptoms. (a) *You may consider the addition of an OAH to INCS. However, scientific evidence shows that adding an OAH to INCS provides no additional benefit over INCS alone. ${ }^{21,45}{ }^{+}$This consensus group does not recommend routine early use of oral corticosteroids (OC); OC use should be reserved for short-term acute severe symptoms. (b) *Evidence for the benefit of these add-ons is lacking. Adapted from BSACI Scadding $2008 .^{1}$ 
7-14 days for control of acute severe symptoms, for example during the peak of the pollen season. If treatment failure continues, the diagnosis should be revisited in the first instance, while surgery or immunotherapy may be subsequent treatment options.

It seems likely that the use of INCS/AZE (single device) may be an additional treatment option prior to immunotherapy. Immunotherapy may be appropriate for some patients with persistent symptoms predominantly due to one allergen such as grass pollen. However, many patients are polysensitised and will remain symptomatic despite immunotherapy; these patients should receive symptomatic treatment in addition to immunotherapy. Relapse following successful immunotherapy treatment may occur; for these patients further treatment options including symptomatic treatment may be considered.

\section{DISCUSSION}

Achieving adequate symptom control is pivotal to successful AR management and may be attained though the stepwise treatment algorithm outlined here. Correct administration technique for INCS and good compliance with treatment regimen are essential to achieve symptom control, and should be checked in the event of early treatment failures. In primary care, following treatment failure on INCS the consensus panel does not recommend increasing the dose of INCS, for which there is little evidence due to the recognised ceiling effect. ${ }^{47}$ In addition, switching INCS is also not recommended as all have comparable efficacy. ${ }^{52-54}$ The panel recommends prescription of INCS/ AZE (single device) as the evidence-based option, ${ }^{46-49}$ rather than adding oral AH to INCS, as there is lack of consistent evidence for the additive efficacy of the latter. $^{21,45}$ The consensus panel judge that positioning INCS/AZE (single device) as a second-line intervention in the primary care pathway may provide value to the health service by averting repeat GP visits (at $£ 45$ per consultation) ${ }^{27}$ or costly secondary care referrals (at approximate costs of $£ 136$ for ENT referral, and $£ 212$ for paediatric referral). ${ }^{55}$ The fundamentals presented in this consensus article should apply in most health-care settings, although obviously different patterns of allergen exposure and allergens will occur, and cost considerations and pharmacotherapy availability may differ.

Achieving adequate symptom control through efficacious prescription medication will avoid patients resorting to selfmedication with add-on over-the-counter (OTC) products, which can lead to adverse effects with prolonged use, e.g., rhinitis medicamentosa with repeat topical nasal decongestants such as oxymetazoline. ${ }^{56}$ There are patients who do not tolerate the firstgeneration oral AHs available OTC (e.g., chlorpheniramine), and the safety of drivers who self-medicate on escalating doses of additive first-generation oral $\mathrm{AHs}$ is a concern. In addition, there is a need for alternatives to OCs for symptom relief for major life events, such as exams and weddings. The panel considered that OCs are positioned too early in the $2008 \mathrm{BSACl}$ algorithm, ${ }^{1}$ and should be recommended only for short-term use to gain control of acute severe symptoms. The forthcoming update to the BSACI guidelines is awaited with interest.

It was noted that all current guidelines take a patient down a route of escalating treatment without taking a step back and assessing whether treatment can be stepped down and when the patient should be referred. Up to $50 \%$ of AR patients in primary care are receiving step 3 treatment with polypharmacy, perhaps inappropriately. ${ }^{27}$ A proportion of patients will not be adequately controlled on initial therapies, and should be referred to secondary care for confirmation of diagnosis and further investigation. They may also receive symptom-specific add-on therapy. There is some advocacy for a step down approach to AR management; for example, starting with INCS/AZE (single device) in patients with previous treatment failure or resistance to monotherapy. After a few weeks of achieving complete control, consideration could be given to treatment reduction e.g., stepping down to INCS alone. However, it is acknowledged that the stepdown approach is based on consensus and more data are needed. Nonetheless, positioning INCS/AZE (single device) first line in secondary care with an integrated check of compliance and technique has the potential to avert costly add-on treatments, repeat visits and possibly the need for immunotherapy or surgery.

\section{CONCLUSION}

With the availability of effective therapy, the majority of AR symptoms can be treated in the primary care setting. This guidance provides a clear pathway for AR treatment in primary care practice that outlines a considered approach to improve the management and control of AR symptoms and improve compliance and patient satisfaction with therapy. Adherence with this care pathway has the potential to limit the cost of providing effective AR management in the UK by avoiding unnecessary treatments, and investigations and avoiding the need for unnecessary repeat GP visits and costly referrals to secondary care in the majority of AR cases.

\section{ACKNOWLEDGEMENTS}

Editorial assistance in preparation of this manuscript was provided by Dr Lisa Buttle and Dr Ruth Murray, of MedScript Ltd. The panel meeting was initiated and funded by Meda.

\section{AUTHOR CONTRIBUTIONS}

All authors attended and contributed at a consensus meeting held in Stirling, Scotland in February 2015. All authors were involved in the drafting of the manuscript, critically revised each draft and gave their final approval for publication. All authors agree to be accountable for all aspects of this work and have participated sufficiently to take public responsibility for the content.

\section{COMPETING INTERESTS}

Brian Lipworth has received unrestricted educational grant support as well as payments for consulting and talks from Meda. Jon Newton has received payment for consulting and talks from Meda. Bhaskar Ram has received support as well as payments for consulting and talks from Meda. lain Small has received payment for consulting and talks from Meda. Jürgen Schwarze has received unrestricted educational grant support as well as payments for consulting from Meda.

\section{REFERENCES}

1. Scadding, G. K. et al. BSACl guidelines for the management of allergic and nonallergic rhinitis. Clin. Exp. Allergy 38, 19-42 (2008).

2. Angier, E., Willington, J., Scadding, G., Holmes, S. \& Walker, S. Management of allergic and non-allergic rhinitis: a primary care summary of the $\mathrm{BSACl}$ guideline. Prim. Care Respir. J. 19, 217-222 (2010).

3. Aberg, N., Hesselmar, B., Aberg, B. \& Eriksson, B. Increase of asthma, allergic rhinitis and eczema in Swedish schoolchildren between 1979 and 1991. Clin. Exp. Allergy 25, 815-819 (1995).

4. Maziak, W. et al. Are asthma and allergies in children and adolescents increasing? Results from ISAAC phase I and phase III surveys in Munster, Germany. Allergy 58, 572-579 (2003).

5. Bousquet, P. J., Demoly, P., Devillier, P., Mesbah, K. \& Bousquet., J. Impact of allergic rhinitis symptoms on quality of life in primary care. Int. Arch. Allergy Immunol. 160, 393-400 (2013).

6. Small, M., Piercy, J., Demoly, P. \& Marsden, H. Burden of illness and quality of life in patients being treated for seasonal allergic rhinitis: a cohort survey. Clin. Transl. Allergy 3, 33 (2013).

7. Walker, S. et al. Seasonal allergic rhinitis is associated with a detrimental effect on examination performance in United Kingdom teenagers: case-control study. J. Allergy Clin. Immunol. 120, 381-387 (2007).

8. Blaiss, M. S. Allergic rhinitis and impairment issues in schoolchildren: a consensus report. Curr. Med. Res. Opin. 20, 1937-1952 (2004). 
9. Szeinbach, S. L., Seoane-Vazquez, E. C., Beyer, A. \& Williams, P. B. The impact of allergic rhinitis on work productivity. Prim. Care Respir. J. 16, 98-105 (2007).

10. Blaiss, M. S. Cognitive, social, and economic costs of allergic rhinitis. Allergy. Asthma. Proc. 21, 7-13 (2000).

11. Lamb, C. E. et al. Economic impact of workplace productivity losses due to allergic rhinitis compared with select medical conditions in the United States from an employer perspective. Curr. Med. Res. Opin. 22, 1203-1210 (2006).

12. Hellgren, J., Cervin, A., Nordling, S., Bergman, A. \& Cardell, L. O. Allergic rhinitis and the common cold-high cost to society. Allergy. 65, 776-783 (2010).

13. de la Hoz, C. B. et al. Allergic rhinitis and its impact on work productivity in primary care practice and a comparison with other common diseases: the Cross-sectional study to evAluate work Productivity in allergic Rhinitis compared with other common diseases (CAPRI) study. Am. J. Rhinol. Allergy 26, 390-394 (2012).

14. Storms, W. Allergic rhinitis-induced nasal congestion: its impact on sleep quality. Prim. Care Respir. J. 17, 7-18 (2008).

15. Nathan, R. A. The burden of allergic rhinitis. Allergy Asthma. Proc. 28, 3-9 (2007).

16. Schatz, M. et al. The burden of rhinitis in a managed care organization. Ann. Allergy Asthma Immunol. 101, 240-247 (2008).

17. Ryan, D., Levy, M., Morris, A., Sheikh, A. \& Walker, S. Management of allergic problems in primary care: time for a rethink? Prim. Care Respir. J. 14, 195-203 (2005).

18. Walker, S., Morton, C. \& Sheikh, A. Diagnosing allergy in primary care: are the history and clinical examination sufficient? Prim. Care Respir. J. 15, 219-221 (2006).

19. Ellis, J., Rafi, I., Smith, H. \& Sheikh, A. Identifying current training provision and future training needs in allergy available for UK general practice trainees: national cross-sectional survey of General Practitioner Specialist Training programme directors. Prim. Care Respir. J. 22, 19-22 (2013).

20. Price, D. et al. UK prescribing practices as proxy markers of unmet need in allergic rhinitis: a retrospective observational study. NPJ Prim. Care Respir. Med. 26, 16033 (2016).

21. Anolik, R., Mometasone Furoate Nasal Spray With Loratadine Study Group. Clinical benefits of combination treatment with mometasone furoate nasal spray and loratadine vs monotherapy with mometasone furoate in the treatment of seasonal allergic rhinitis. Ann. Allergy Asthma Immunol. 100, 264-271 (2008).

22. Esteitie, R., deTineo, M., Naclerio, R. M. \& Baroody, F. M. Effect of the addition of montelukast to fluticasone propionate for the treatment of perennial allergic rhinitis. Ann. Allergy Asthma Immunol. 105, 155-161 (2010).

23. Bousquet, J. et al. Allergic Rhinitis and its Impact on Asthma (ARIA) 2008 update (in collaboration with the World Health Organization, GA(2)LEN and AllerGen). Allergy. 63, 8-160 (2008).

24. Scadding, G. \& Walker, S. Poor asthma control?-then look up the nose. The importance of co-morbid rhinitis in patients with asthma. Prim. Care Respir. J. 21, 222-228 (2012).

25. Bachert, C. et al. Allergic rhinitis, rhinosinusitis, and asthma: one airway disease. Immunol. Allergy Clin. North. Am. 24, 19-43 (2004).

26. Clatworthy, J., Price, D., Ryan, D., Haughney, J. \& Horne, R. The value of self-report assessment of adherence, rhinitis and smoking in relation to asthma control. Prim. Care Respir. J. 18, 300-305 (2009).

27. Price, D. et al. The hidden burden of adult allergic rhinitis: UK healthcare resource utilisation survey. Clin. Exp. Allergy 5, 39 (2015).

28. Azevedo, P. et al. Control of allergic rhinitis and asthma test (CARAT): dissemination and application in primary care. Prim. Care Respir. J. 22, 112-116 (2013).

29. Nathan, R. A. The rhinitis control assessment test: implications for the present and future. Curr. Opin. Allergy Clin. Immunol. 14, 13-19 (2014).

30. Nathan, R. A. et al. Qualitative development of the Rhinitis Control Assessment Test (RCAT), an instrument for evaluating rhinitis symptom control. Patient $\mathbf{3}$, 91-99 (2010).

31. Demoly, P. et al. Assessment of disease control in allergic rhinitis. Clin. Transl. Allergy 3, 7 (2013).

32. Bousquet, J. et al. MACVIA clinical decision algorithm in adolescents and adults with allergic rhinitis. J. Allergy. Clin. Immunol. 138, 367-374 (2016).

33. Meltzer, E. O. et al. Burden of allergic rhinitis: results from the Pediatric Allergies in America survey. J. Allergy Clin. Immunol. 124, S43-S70 (2009).

34. Hellings, P. W. et al. Explorative study on patient's perceived knowledge level, expectations, preferences and fear of side effects for treatment for allergic rhinitis. Clin. Transl. Allergy 2, 9 (2012).

35. Ciprandi, G. et al. Patient-related factors in rhinitis and asthma: the satisfaction with allergy treatment survey. Curr. Med. Res. Opin. 27, 1005-1011 (2011).
36. Gronhoj, L. C., Gyldenlove, M. \& Linneberg, A. Allergic rhinitis is often undiagnosed and untreated: results from a general population study of Danish adults. Clin. Respir. J. 7, 354-358 (2013).

37. Ryan, D. et al. Management of allergic rhinitis in UK primary care: baseline audit. Prim. Care. Respir. J. 14, 204-209 (2005).

38. Garavello, W., Di, B. F., Romagnoli, M., Sambataro, G. \& Gaini, R. M. Nasal rinsing with hypertonic solution: an adjunctive treatment for pediatric seasonal allergic rhinoconjunctivitis. Int. Arch. Allergy Immunol. 137, 310-314 (2005).

39. Tomooka, L. T., Murphy, C. \& Davidson, T. M. Clinical study and literature review of nasal irrigation. Laryngoscope. 110, 1189-1193 (2000).

40. Horak, F. \& Zieglmayer, U. P. Azelastine nasal spray for the treatment of allergic and nonallergic rhinitis. Expert. Rev. Clin. Immunol. 5, 659-669 (2009).

41. Ellis, A. K., Zhu, Y., Steacy, L. M., Walker, T. \& Day, J. H. A four-way, double-blind, randomized, placebo controlled study to determine the efficacy and speed of azelastine nasal spray, versus loratadine, and cetirizine in adult subjects with allergen-induced seasonal allergic rhinitis. Allergy. Asthma. Clin. Immunol. 9, 16 (2013).

42. Erdogan, B. A., Sanli, A., Paksoy, M., Altin, G. \& Aydin, S. Quality of life in patients with persistent allergic rhinitis treated with desloratadine monotherapy or desloratadine plus montelucast combination. Kulak. Burun. Bogaz. Ihtis. Derg. 24, 217-224 (2014).

43. Cingi, C. et al. Desloratadine-montelukast combination improves quality of life and decreases nasal obstruction in patients with perennial allergic rhinitis. Int. Forum. Allergy Rhinol. 3, 801-806 (2013).

44. Dymista summary of product characteristics. https://www medicines org uk/emc/ medicine/27579 2014. [last accessed 02.09.16]

45. Di Lorenzo, G. et al. Randomized placebo-controlled trial comparing fluticasone aqueous nasal spray in mono-therapy, fluticasone plus cetirizine, fluticasone plus montelukast and cetirizine plus montelukast for seasonal allergic rhinitis. Clin. Exp. Allergy 34, 259-267 (2004).

46. Carr, W. et al. A novel intranasal therapy of azelastine with fluticasone for the treatment of allergic rhinitis. J. Allergy Clin. Immunol. 129, 1282-1289 (2012).

47. Meltzer, E. et al. Clinically relevant effect of a new intranasal therapy (MP29-02) in allergic rhinitis assessed by responder analysis. Int. Arch. Allergy Immunol. 161, 369-377 (2013)

48. Price, D. et al. A new therapy (MP29-02) is effective for the long-term treatment of chronic rhinitis. J. Investig. Allergol. Clin. Immunol. 23, 495-503 (2013).

49. Berger, W. E. et al. Long-term, randomized safety study of MP29-02 (a novel intranasal formulation of azelastine hydrochloride and fluticasone propionate in an advanced delivery system) in subjects with chronic rhinitis. J. Allergy Clin. Immunol. Pract. 2, 179-185 (2014).

50. Klimek, L. et al. MP-AzeFlu provides rapid and effective allergic rhinitis control in real-life: a pan-European study. Allergy Asthma Proc. 37, 376-386 (2016).

51. D'Addio, A. et al. Deposition characterisitcs of a new allergic rhinitis nasal spray (MP29-02) in an anatomical model of the human nasal cavity. Allergy. 69, A842 (2014).

52. Aneeza, W. H. et al. Efficacy of mometasone furoate and fluticasone furoate on persistent allergic rhinoconjunctivitis. Allergy Rhinol (Providence) 4, e120-e126 (2013).

53. Okubo, K., Nakashima, M., Miyake, N., Komatsubara, M. \& Okuda, M. Comparison of fluticasone furoate and fluticasone propionate for the treatment of Japanese cedar pollinosis. Allergy Asthma Proc. 30, 84-94 (2009).

54. Mandl, M., Nolop, K. \& Lutsky, B. N. Comparison of once daily mometasone furoate (Nasonex) and fluticasone propionate aqueous nasal sprays for the treatment of perennial rhinitis. 194-079 Study Group. Ann. Allergy Asthma Immunol. 79, 370-378 (1997).

55. Unit costs of health and social care. https://www.gov.uk/government/publications/ national-tariff-payment-system-2014-to-2015. [last accessed 10.11.15]

56. Graf, P. Rhinitis medicamentosa: a review of causes and treatment. Treat. Respir. Med. 4, 21-29 (2005).

This work is licensed under a Creative Commons Attribution 4.0 International License. The images or other third party material in this article are included in the article's Creative Commons license, unless indicated otherwise in the credit line; if the material is not included under the Creative Commons license, users will need to obtain permission from the license holder to reproduce the material. To view a copy of this license, visit http://creativecommons.org/licenses/by/ $4.0 /$

(c) The Author(s) 2017 